\title{
EXACT STEADY STATES OF DISORDERED HOPPING PARTICLE MODELS WITH PARALLEL AND ORDERED SEQUENTIAL DYNAMICS
}

\author{
M. R. Evans"] \\ Department of Physics and Astronomy \\ University of Edinburgh \\ Mayfield Road, Edinburgh EH9 3JZ, U.K.
}

\begin{abstract}
A one-dimensional driven lattice gas with disorder in the particle hopping probabilities is considered. It has previously been shown that in the version of the model with random sequential updating, a phase transition occurs from a low density inhomogeneous phase to a high density congested phase. Here the steady states for both parallel (fully synchronous) updating and ordered sequential updating are solved exactly and the phase transition shown to persist in both cases. For parallel dynamics and forward ordered sequential dynamics the phase transition occurs at the same density but for backward ordered sequential dynamics it occurs at a higher density. In both cases the critical density is higher than that for random sequential dynamics. In all the models studied the steady state velocity is related to the fugacity of a Bose system suggesting a principle of minimisation of velocity. A generalisation of the dynamics where the hopping probabilities depend on the number of empty sites in front of the particles, is also solved exactly in the case of parallel updating. The models have natural interpretations as simplistic descriptions of traffic flow. The relation to more sophisticated traffic flow models is discussed.
\end{abstract}

Date: 29/4/1997 Submitted to Journal of Physics A.

Key words: $\quad$ asymmetric exclusion process, random rates, steady state, parallel dynamics, phase transition, Bose condensation, traffic flow

PACS numbers: $05.40+\mathrm{j}, 05.60+\mathrm{w}, 64.60 \mathrm{Cn}, 89.40+\mathrm{k}$

Short Title: Disordered Hopping Particle Models

\footnotetext{
${ }^{1}$ Royal Society University Research Fellow
} 


\section{Introduction}

The asymmetric simple exclusion process (ASEP) is an archetypal example of a driven diffusive system [1, 2] for which analytical results are possible, particularly in one dimension [3]. The model comprises particles which hop stochastically in a preferred direction with hard core exclusion imposed. The model has a natural interpretation as a simplistic description of traffic flow on a one lane road and indeed forms the basis for more sophisticated traffic

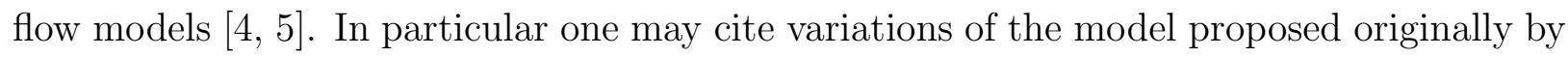
Nagel and Schreckenberg [6, 0, 8, 9, 10, 11].

However, a basic difference between the original ASEP and traffic flow models lies in the updating scheme. In the mathematical literature the ASEP is usually defined in continuous time or, equivalently for the purposes of simulation, by a random sequential updating scheme where for each update a particle is selected at random. In contrast, when simulating traffic flow parallel updating is usually employed for reasons both idealistic - parallel dynamics provides a perhaps more faithful representation of real traffic - and pragmatic - parallel dynamics yields economy of random numbers.

For random sequential dynamics a relative wealth of exact results on the ASEP are now available [2, 3, 13, 14, 15, 16, 17, 18, 19, in particular the steady states of various models have been constructed using a matrix product ansatz [3, 14, 20, 21, 22, 23, 24]. This technique has been extended to a sublattice parallel updating scheme [25, 26, 27] and, in the case of open boundary conditions, to an ordered sequential scheme [28, 29]. However, for fully parallel dynamics only a few exact results are known [8, 30].

In the present work we determine exactly the steady state of an ASEP with disorder for both parallel dynamics and ordered sequential dynamics, in the case of periodic boundary conditions. The disorder takes the form of quenched random hopping probabilities assigned to each particle. Other types of disorder and inhomogeneities such as random rates associated with lattice sites [32], slow defect sites [31, 33, 27] or slow defect particles [34, 35] have been considered. The random sequential version of the disordered model considered here has previously been studied [36, 37, 38] and a transition shown to occur between a low density inhomogeneous phase, where a tailback forms behind the slowest particle, to a high density congested phase.

In the present work it will be demonstrated that this transition persists under parallel and ordered sequential updating. The difficulty in obtaining exact results for parallel dynamics

and ordered sequential dynamics lies in the construction of the transfer matrix. Using a technique inspired by ref. [39] we explicitly construct the transfer matrices in a convenient form that allows the steady states to be demonstrated.

From the point of view of traffic flow the phase structure of the disordered model is of interest since the disorder induces emergent jams at low densities, whereas in other traffic 
flow models where a phase transition occurs it is in the high density phase that the jams emerge [9, 11, 12]. The coarsening of the resulting jams has also been studied [40, 10, 38, 41]. From a theoretical viewpoint it was shown in the random sequential case that the transition has a strong analogy with Bose condensation [37 and that the steady state velocity of a particle was equivalent to the fugacity of an ideal Bose gas. This analogy will be pursued here for the parallel and ordered sequential cases and it will be shown that the steady state velocity remains related to the fugacity of a Bose system.

It is of interest to determine whether distinct updating schemes can produce different behaviour. It turns out that the the value of the critical density may depend on the updating scheme. It will be shown that the critical density is highest, implying the throughput most efficient, for a backward ordered updating scheme where the updating sequence is opposite to the direction of flow of particles. In contrast, the random sequential updating scheme yields the lowest critical density.

Another key difference between traffic models (e.g. the model of [6]) and the ASEP is that in traffic models particles may move a distance greater than one lattice spacing, thus implying that the dynamics is not nearest neighbour in the sense that cars are aware of cars several lattice sites ahead. At present analytical results are not generally available for the case of hopping more than one lattice site, although a step towards this goal has been made [30. On the other hand dynamics of range greater than nearest neighbour may be mimicked by letting the probability with which a particle hops forward depend on the number of empty lattice sites in front of it. Indeed, the concept of a braking distance furnishes a natural interpretation for hopping probabilities which increase as a function of the empty space in front.

In the present work we solve the steady state of a generalisation of the dynamics where the disordered hopping probabilities are dependent on the empty space in front, restricting our attention to parallel updating.

The paper is organised as follows. In section 2 the model and updating schemes considered are defined and exact expressions for the steady states presented. The proof of these expressions is somewhat technical and is deferred until section 4. Prior to that, in section 3, the phase transition and the analogy with Bose condensation is analysed. In section 5 the generalisation of the model to the case of space dependent hopping probabilities is considered and in section 6 conclusions are drawn. 


\section{Model Definitions and Steady States}

In this work we study asymmetric exclusion models where the particle hopping probabilities are quenched random variables. We consider $M$ particles labelled $\{\mu=1, \ldots, M\}$ hopping on a one dimensional lattice of size $N$ sites labelled $\{i=1, \ldots, N\}$ with periodic boundary conditions ( site $N+i=$ site $i$ ). The random sequential version of the model was considered in [37. We now define three distinct variants of the model according to the following dynamics.

Parallel Dynamics: at each time-step all particles $\{\mu=1, \ldots, M\}$ simultaneously attempt to hop forward each with its own probability $p_{\mu}$. A hop is only carried out if the target site was empty before the update. Since no backwards hops are permitted the question of what would happen if two particles simultaneously attempted to hop onto the same site does not arise.

Ordered Sequential Dynamics: in this case a time-step corresponds to updating each particle in a fixed sequence. At each update the relevant particle $\mu$ attempts a hop forward with probability $p_{\mu}$. We consider two sequences for the updating

Forward updating in which the order is $1,2, \ldots M$.

Backward updating in which the order is $M, M-1, \ldots 1$

Since particles cannot overtake, the sequence of particles is preserved in all three variants.

\section{1. Expressions for the steady states}

In order to express the steady state of each variant of the model defined above, we consider the weight $F\left(n_{1}, n_{2}, \ldots, n_{M}\right)$ of a configuration comprising particle 1 followed by $n_{1}$ holes (empty lattice sites); particle 2 followed by $n_{2}$ holes and so on. The weights are related to probabilities $P_{N}\left(\left\{n_{\mu}\right\}\right)$ via a normalisation $Z_{N, M}$ defined by

$$
P_{N}\left(\left\{n_{\mu}\right\}\right)=F\left(\left\{n_{\mu}\right\}\right) / Z_{N, M} .
$$

We now present exact expressions (to be proven in later sections) for the steady state of the three variants. In each case the steady state weights have the factorised form

$$
F\left(\left\{n_{\mu}\right\}\right)=\prod_{\mu=1}^{M} f_{\mu}\left(n_{\mu}\right)
$$


The different variants have different expressions for $f_{\mu}\left(n_{\mu}\right)$ as follows.

\section{Parallel Dynamics}

In this case

$$
\begin{aligned}
f_{\mu}\left(n_{\mu}\right) & =\left(1-p_{\mu}\right) \text { for } n_{\mu}=0 \\
& =\left(\frac{1-p_{\mu}}{p_{\mu}}\right)^{n_{\mu}} \text { for } n_{\mu}>0
\end{aligned}
$$

which may be rewritten employing the usual Heaviside function as

$$
f_{\mu}\left(n_{\mu}\right)=\left(1-p_{\mu}\right)+\left[\left(\frac{1-p_{\mu}}{p_{\mu}}\right)^{n_{\mu}}-\left(1-p_{\mu}\right)\right] \theta\left(n_{\mu}\right) .
$$

Note that the pure case, which has $p_{\mu}=p \forall \mu$ recovers a result obtained in [8] through a combinatorial argument.

\section{Forward Ordered Sequential Dynamics}

To define a steady state for ordered sequential dynamics one must specify the point in the update sequence to which the steady state refers. For forward updating we define the steady state as that after the update of particle $M$ (the final update of the sequence). That is, the steady state weights are those for finding the system in a given configuration after the last update of a sequence, and before the first update of the next sequence. In this case

$$
\begin{aligned}
f_{\mu}\left(n_{\mu}\right) & =\left(1-p_{\mu}\right)+\left[\left(\frac{1-p_{\mu}}{p_{\mu}}\right)^{n_{\mu}}-\left(1-p_{\mu}\right)\right] \theta\left(n_{\mu}\right) \text { for } \mu \neq M \\
f_{M}\left(n_{M}\right) & =\left(\frac{1-p_{M}}{p_{M}}\right)^{n_{M}}
\end{aligned}
$$

\section{Backward Ordered Sequential Dynamics}

In this case the steady state again refers to the weight for finding the system in a given configuration after the final update of the sequence, this time the update of particle 1.

$$
\begin{aligned}
f_{\mu}\left(n_{\mu}\right) & =\left(\frac{1-p_{\mu}}{p_{\mu}}\right)^{n_{\mu}} \text { for } \mu \neq M \\
f_{M}\left(n_{M}\right) & =\left(1-p_{M}\right)+\left[\left(\frac{1-p_{M}}{p_{M}}\right)^{n_{M}}-\left(1-p_{M}\right)\right] \theta\left(n_{M}\right)
\end{aligned}
$$

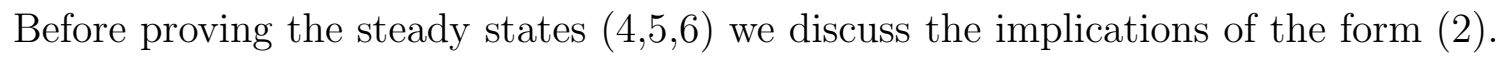




\section{Phase transition and analogy with Bose condensation}

A steady state of the factorised form (2) gives rise to the possibility of a phase transition. In 37 the analogy with Bose condensation was made. Let us review briefly the qualitative aspects of the phase transition and the analogy.

The two phases exhibited by the model are a congested phase that exists at high density and an inhomogeneous phase that exists at low density. In the congested phase, the velocity of particles is limited by the availability of empty sites, whereas in the inhomogeneous phase the velocity is limited by the hopping rate of the slowest particle. Thus, the inhomogeneous phase may be pictured as comprising two regions: a tailback behind the slowest particle (a high density region) and empty space in front of the slowest particle (a low density region). The analogy with Bose condensation is to think of the empty sites as bosons and the state of a boson as determined by which car it is immediately in front of. Then in the congested phase the bosons are thinly spread over all the Bose states (i.e. there are uniformly small gaps between the particles) whereas in the inhomogeneous phase the bosons are condensed in front of the slowest particle (there is a large empty space in front of the slowest particle).

Before exploring the analogy with Bose condensation we shall discuss the mathematics of how calculations are performed. We focus first on the case of parallel dynamics and extend the results to ordered sequential dynamics in section 3.3.

To calculate quantities of interest, in particular steady state averages, one must first have at hand expressions for the normalisation $Z_{N, M}$ of the weights (2) defined through (11). Due to the constraint of $N-M$ holes the normalisation may be written

$$
Z_{N, M}=\sum_{n_{1}, n_{2} \ldots n_{M}} \delta_{\sum_{\mu} n_{\mu},(N-M)} \prod_{\mu=1}^{M} f_{\mu}\left(n_{\mu}\right)
$$

This quantity may be considered as the canonical partition function of a thermodynamic system and in the standard way [42] it may be written using an integral representation of the delta function as

$$
Z_{N, M}=\oint \frac{d z}{2 \pi i} z^{-(N-M+1)} \mathcal{Z}(z),
$$

where the grand canonical partition function $\mathcal{Z}$ is given by

$$
\mathcal{Z}(z)=\prod_{\mu=1}^{M}\left[\sum_{n_{\mu}=0}^{\infty} z^{n_{\mu}} f_{\mu}\left(n_{\mu}\right)\right] .
$$

For large $N, M(8)$ is dominated by the saddle point of the integral and $z^{*}$, the value of $z$ at the saddle point, is the fugacity. 
We now calculate the velocity $v$, defined as the steady state average of the rate of hopping of a given particle. Since the particles cannot overtake each other, the velocity is the same for any particle $\mu$. Taking $\mu=1$ one finds

$$
\begin{aligned}
v & =Z_{N, M}^{-1} \oint \frac{d z}{2 \pi i} z^{-(N-M+1)}\left[p_{1} \sum_{n_{1}=1}^{\infty} z^{n_{1}} f_{1}\left(n_{1}\right)\right] \prod_{\mu=2}^{M}\left[\sum_{n_{\mu}=0}^{\infty} z^{n_{\mu}} f_{\mu}\left(n_{\mu}\right)\right] \\
& \simeq\left[p_{1} \sum_{n_{1}=1}^{\infty}\left(z^{*}\right)^{n_{1}} f_{1}\left(n_{1}\right)\right]\left[\sum_{n_{1}=0}^{\infty}\left(z^{*}\right)^{n_{1}} f_{1}\left(n_{1}\right)\right]^{-1} \quad \text { for large } N, M \\
& =\frac{z^{*}}{1+z^{*}},
\end{aligned}
$$

where the last equality results from performing the geometric series obtained when (4) is inserted in (11).

In the following the thermodynamic limit is defined by

$$
N \rightarrow \infty \text { with } \quad M=\rho N,
$$

with the density $\rho$ held fixed. In order to determine the fugacity one uses the condition

$$
N-M=z \frac{\partial \ln \mathcal{Z}}{\partial z}
$$

which is the saddle point condition for (8) (or equivalently the condition that in the grand canonical ensemble the average number of empty sites is $N-M$ ). Using (四) one finds that (9) becomes

$$
\mathcal{Z}(z)=\prod_{\mu=1}^{M}\left[1-p_{\mu}+\frac{\left(1-p_{\mu}\right) z}{p_{\mu}-\left(1-p_{\mu}\right) z}\right]
$$

and (14) yields

$$
N-M=\frac{z}{1+z} \sum_{\mu=1}^{M} \frac{1}{p_{\mu}-\left(1-p_{\mu}\right) z}
$$

Now using the relation between $z^{*}$ and $v(12)$, one obtains in the thermodynamic limit

$$
1-\rho=v(1-v) \frac{1}{N} \sum_{\mu=1}^{M} \frac{1}{p_{\mu}-v}
$$

\section{1. The disorder average}

In the following we shall consider the particle hopping probabilities $p_{\mu}$ as quenched random variables drawn from a common distribution 38

$$
\mathcal{P}(p)=\frac{(\gamma+1)}{(1-c)^{\gamma+1}}(p-c)^{\gamma}
$$


with support on the interval $[c, 1)$. Other distributions may also be considered [37], but the qualitative behaviour is determined by the power $\gamma$.

For the disordered system one wishes to obtain properties given by a typical realisation of the disorder (here the particle hopping probabilities), for example the typical velocity. Usually in the theory of disordered systems one expects quantities such as $v$ to self-average but quantities exponentially large in the system size, such as $Z_{N, M}$, not to. That is, one expects as $N \rightarrow \infty, v \rightarrow \bar{v}$ with probability one, where the bar indicates an average over the particle hopping probabilities. When working in the grand canonical ensemble one has directly an equation for $v$ in the thermodynamic limit, (17). Thus, with the assumption that $v$ is self-averaging, one simply has to average (17) to obtain an equation for the typical velocity.

Now, in the thermodynamic limit (13), the fraction of particles with hopping probabilities between $p$ and $p+d p$ converges to $\mathcal{P}(p) d p$. Equally the velocity of the slowest particle converges to $c$. For the sake of clarity, however, it is convenient to assume that the slowest particle, taken to be particle 1 , has velocity exactly equal to $c$. Therefore (17) may be replaced by

$$
1-\rho=\rho(1-v) I(v)+\frac{\left\langle n_{1}\right\rangle}{N}
$$

where

$$
I(v)=\int_{c}^{1} d p \mathcal{P}(p) \frac{v}{p-v}
$$

Since the rhs of (16) is an increasing function of $z$ we deduce that the rhs of (17) is an increasing function of $v$. However, $z$ was introduced in (8) as a contour integration variable therefore the saddle point $z^{*}$ must lie between 0 and any pole in the integrand of (8). Owing to this, the maximum value $z$ can take is $c /(1-c)$ and the maximum value of $v$ is $c$.

If the integral (20) diverges as $v \rightarrow c$ then (19) can always be satisfied with $v<c$ and $<n_{1}>/ N$ zero. However, it turns out that for the distribution (18) with $\gamma>0, I(v)$ is always finite as $v \rightarrow c$ from below. Therefore when

$$
I(c)<\frac{(1-\rho)}{\rho(1-c)},
$$

which holds for $\rho$ below a critical value $\rho_{c}$, (19) can only be satisfied with $v \rightarrow c$ and $<n_{1}>/ N$ non-zero. As (19) expresses the constraint in the number of holes, we see that in this case a finite fraction of the holes must reside in front of the slowest particle. Thus, we have the inhomogeneous low density phase. On the other hand for $\rho>\rho_{c}$, (19) may be satisfied for $v<c$ and $\left\langle n_{1}>/ N\right.$ zero, in which case we have the high density congested phase.

By this point the qualitative analogy with Bose condensation should be apparent. However, we first remark that the exact mapping onto an ideal Bose gas found for random 
sequential dynamics [16, 37] no longer holds. In the mapping $n_{\mu}$ is viewed as the occupation number of the $\mu^{\text {th }}$ Bose state; applying this to Eq. 4 one sees that in the parallel update system an unoccupied Bose state is penalised. This effective repulsion between particles already occurs in the pure case [8]. Secondly, for random sequential dynamics the velocity was exactly equivalent to the fugacity of an ideal Bose gas [37], whereas in the present case although $v$ is still an increasing function of $z^{*}$, the relation is modified to (12). Nevertheless, the analogy with Bose condensation remains useful. The slowest particle corresponds to the Bose ground state and the distribution of particle hopping probabilities $\mathcal{P}(p)$ corresponds to the density of states of the Bose gas. Thus the transition to the inhomogeneous phase corresponds to a condensation of a finite fraction of bosons into the Bose ground state (i.e. a finite fraction of the holes reside in front of the slowest particle).

\section{2. Critical Behaviour}

In order to analyse the phase transition we require at our disposal the asymptotic behaviour of (19) as $v \rightarrow c$. The expansion in $\epsilon$ where

$$
\epsilon=\ln \left[\frac{c}{v}\right] \simeq \frac{c-v}{c} \text { as } v \rightarrow c
$$

is carried out in appendix A. The result is

$$
\begin{aligned}
& \text { For } \gamma<1 \quad I(v)=\frac{(1+\gamma) c}{\gamma(1-c)}+(1+\gamma)\left(\frac{c}{1-c}\right)^{1+\gamma} \Gamma(1+\gamma) \Gamma(-\gamma) \epsilon^{\gamma}+\ldots \\
& \text { For } \gamma=1 \quad I(v)=\frac{2 c}{(1-c)}+2\left(\frac{c}{1-c}\right)^{2} \epsilon \ln (\epsilon)+\ldots \\
& \text { For } \gamma>1 \quad I(v)=\frac{(1+\gamma) c}{\gamma(1-c)}-\frac{(1+\gamma) c}{\gamma(1-c)}\left[1+\frac{c}{(1-c)} \frac{\gamma}{(\gamma-1)}\right] \epsilon+\ldots
\end{aligned}
$$

we see from $(23-25)$ that $I(c)=(1+\gamma) c /(\gamma(1-c))$ which implies, together with the condition for the transition (21), that the critical value of $\rho$ is

$$
\rho_{c}=\frac{\gamma}{\gamma+c+\gamma c}
$$

One should note that for that $\gamma=0$ (a flat distribution of hopping probabilities) the transition to a congested phase occurs at zero density.

As $\rho$ is increased above the critical value the velocity decreases from $c$ according to

$$
\begin{aligned}
& \text { For } \gamma<1 \quad v \simeq c-\left[\frac{(1-c)^{\gamma}}{\rho_{c}^{2} c \Gamma(1+\gamma)|\Gamma(-\gamma)|}\right]^{1 / \gamma}\left(\rho-\rho_{c}\right)^{1 / \gamma}+\ldots \\
& \text { For } \gamma=1 \quad v \simeq c-\frac{(1-c)}{2 \rho_{c}^{2} c} \frac{\left(\rho-\rho_{c}\right)}{\left|\ln \left(\rho-\rho_{c}\right)\right|}+\ldots \\
& \text { For } \gamma>1 \quad v \simeq c-\frac{(1-c) \gamma(\gamma-1)}{\rho_{c}^{2}(1+\gamma)[c+(\gamma-1)(1-c)]}\left(\rho-\rho_{c}\right)+\ldots
\end{aligned}
$$


One can also consider the current or throughput $J$ defined by

$$
J=\rho v .
$$

In the inhomogeneous phase $\left(\rho<\rho_{c}\right)$ the current increases linearly with $\rho$ and $J=c \rho$. Using the expansions (27 29) the behaviour of the current in the high density congested phase can be analysed. Just above the transition the current increases with $\rho$ if $\gamma<\gamma^{*}$ but decreases if $\gamma>\gamma^{*}$ where

$$
\gamma^{*}=\frac{1-c+c^{2}}{2(1-c)}\left[1+\sqrt{1+4(1-c) c^{2} /\left(1-c+c^{2}\right)^{2}}\right] .
$$

Thus the maximum current is achieved in the high density phase if $\gamma<\gamma^{*}$ and exactly at the phase transition if $\gamma>\gamma^{*}$. However it does not appear that $\gamma^{*}$ is universal because it depends on $c$ i.e. it depends on the particular choice of $\mathcal{P}(p)$.

\section{3. Ordered Sequential Dynamics}

For forward sequential or backward sequential dynamics the velocity may be defined as the probability that the first particle in the updating sequence hops forward. It is easy to check that (12) is still the correct expression for the velocity in both these cases so that the velocity is always related to the fugacity in a simple way. By performing the sums in (9) one obtains for forward updating

$$
\mathcal{Z}(z)=\prod_{\mu=1}^{M-1}\left[1-p_{\mu}+\frac{\left(1-p_{\mu}\right) z}{p_{\mu}-\left(1-p_{\mu}\right) z}\right]\left[\frac{p_{M}}{p_{M}-\left(1-p_{M}\right) z}\right] .
$$

and for backward updating

$$
\mathcal{Z}(z)=\prod_{\mu=1}^{M-1}\left[\frac{p_{\mu}}{p_{\mu}-\left(1-p_{\mu}\right) z}\right]\left[1-p_{M}+\frac{\left(1-p_{M}\right) z}{p_{M}-\left(1-p_{M}\right) z}\right] .
$$

For forward updating the saddle point value of the fugacity $z$ and hence the velocity will be the same as as for parallel updating, implying the same critical density. However,

for backward updating (33) gives a different saddle point value of $z$ to (15), and it can be checked that (19) is modified to

$$
\left(\frac{1-\rho}{\rho}\right)=(1-v) I(v)-v,
$$

giving

$$
\rho_{c}=\frac{\gamma}{c+\gamma}
$$


We see that for backward updating $\rho_{c}$ is greater than for parallel and forward updating which share the same $\rho_{c}$ given by $(26)$. Since the velocity in the low density phase is greater than that in the high density phase, this means that backward updating yields the best throughput. One might expect this because backward updating increases the chance that the site in front of the next particle to be updated has just been vacated. One can also calculate the critical density for random sequential dynamics [37, 38] to find $\rho_{c}=\gamma(1-c) /(c+\gamma)$ which is lower than both (35) and (26). This implies that random sequential updating gives the poorest throughput.

\section{Proof of Steady State for Ordered Sequential and Parallel Dynamics}

In all three variants of the model considered here the updating rules comprises a determined time-step. Therefore a transfer matrix may be used to express the condition for the steady state weights as

$$
\sum_{\mathcal{C}^{\prime}} T\left(\mathcal{C}, \mathcal{C}^{\prime}\right) F\left(\mathcal{C}^{\prime}\right)=F(\mathcal{C})
$$

where $T\left(\mathcal{C}, \mathcal{C}^{\prime}\right)$ are the components of the transfer matrix. In (36) $T\left(\mathcal{C}, \mathcal{C}^{\prime}\right)$ is the probability of going from configuration $\mathcal{C}^{\prime}$ to $\mathcal{C}$ in one time-step and $T(\mathcal{C}, \mathcal{C})$ is the probability of remaining in configuration $\mathcal{C}$ after a time-step. A configuration is specified by the hole occupation numbers $\mathcal{C}=\left\{n_{1}, \ldots, n_{M}\right\}$. We see from (36) that the steady state weights form an eigenvector of the transfer matrix with eigenvalue one.

In the construction of the transfer matrix that follows, it is convenient to use an operator notation. To each configuration $\left\{n_{1}, \ldots, n_{M}\right\}$ is associated a vector $\left|n_{1}, \ldots, n_{M}\right\rangle$, to be referred to as the configuration vector. The configuration vectors $\left|n_{1}, \ldots, n_{M}\right\rangle$ form an orthonormal basis for the vector space of configurations. We write the weight of a configuration as

$$
F\left(n_{1}, \ldots, n_{\mu}\right)=\left\langle F \mid n_{1}, \ldots, n_{\mu}\right\rangle
$$

Thus, the the weight of a configuration $\left\{n_{1}, \ldots, n_{M}\right\}$ is the component in the direction $\left\langle n_{1}, \ldots, n_{M}\right|$ of the bra vector $\langle F|$.

With this notation we may rewrite (36) as

$$
\left\langle F|\hat{T}| n_{1}, \ldots, n_{\mu}\right\rangle=\left\langle F \mid n_{1}, \ldots, n_{\mu}\right\rangle
$$

where $\hat{T}$ is an operator acting on the space of configuration vectors defined above: $\hat{T}$ acting on a given configuration vector generates the possible configuration vectors before an update multiplied by the appropriate transition probabilities. We shall refer to $\hat{T}$ as the transfer matrix as well as its components $T\left(\mathcal{C}, \mathcal{C}^{\prime}\right)$. Our task now is to construct $\hat{T}$. 


\section{1. Construction of the Transfer Matrices}

In this subsection we construct the transfer matrix for all three variants of the dynamics. The desired form is of a trace of a product of matrices, each of which contains as elements operators acting at the relevant site [39]. The technique is most directly illustrated in the case of ordered sequential dynamics, which we consider first in this subsection.

The transfer matrix for a full time-step of ordered sequential dynamics may be written as an ordered product of $M$ operators $h_{\mu-1 \mu}$ corresponding to the update of each particle $\mu$ in sequence. Recalling that $\hat{T}$ acting on $\left|n_{1}, \ldots, n_{\mu}\right\rangle$ generates the possible configurations leading to $\left|n_{1}, \ldots, n_{\mu}\right\rangle$, one deduces that to correctly generate these configurations we first act on $\left|n_{1}, \ldots, n_{\mu}\right\rangle$ with an operator corresponding to the last update of the sequence, then with an operator corresponding to the second last update and so on, so that for the forward updating

$$
\hat{T}_{F}=\prod_{\mu=1}^{M} h_{\mu-1 \mu} .
$$

By similar reasoning, for backward updating the transfer matrix $T_{B}$ is

$$
\hat{T}_{B}=\prod_{\mu=1}^{M} h_{M-\mu M-\mu+1} .
$$

In both cases the operator $h_{\mu-1 \mu}$ is given by

$$
h_{\mu-1 \mu}=\mathbb{1}-p_{\mu} a_{\mu} a_{\mu}^{\dagger}+p_{\mu} a_{\mu-1}^{\dagger} a_{\mu}
$$

where $a_{\mu}$ is a raising operator and $a_{\mu}^{\dagger}$ is a lowering operator acting on the vector space spanned by $\mid n_{1}, \ldots, n_{M}>$ :

$$
\begin{aligned}
a_{\mu} \mid n_{1}, \ldots, n_{\mu}, \ldots, n_{M}> & =\mid n_{1}, \ldots, n_{\mu}+1, \ldots, n_{M}> \\
a_{\mu}^{\dagger} \mid n_{1}, \ldots, n_{\mu}, \ldots, n_{M}> & =\mid n_{1}, \ldots, n_{\mu}-1, \ldots, n_{M}>\text { if } n_{\mu}>0 \\
& =0 \text { if } n_{\mu}=0
\end{aligned}
$$

and one may verify that

$h_{\mu-1 \mu}\left|n_{1}, \ldots, n_{M}>=\left(1-p_{\mu} \theta\left(n_{\mu}\right)\right)\right| n_{1}, \ldots, n_{M}>+p_{\mu} \theta\left(n_{\mu-1}\right) \mid n_{1}, \ldots, n_{\mu-1}-1, n_{\mu}+1, \ldots, n_{M}>$,

correctly giving the configurations leading to $\mid n_{1}, \ldots, n_{M}>$ after the update of particle $\mu$.

To proceed further we employ techniques used in the study of integrable models [39]. First it is easy to check that (41) may be rewritten in two ways as

$$
\begin{aligned}
h_{\mu-1 \mu} & =\left(\mathbb{1}, a_{\mu-1}^{\dagger}\right)\left(\begin{array}{c}
\mathbb{1}-p_{\mu} a_{\mu} a_{\mu}^{\dagger} \\
p_{\mu} a_{\mu}
\end{array}\right) \\
& =\left(\mathbb{1}-p_{\mu} a_{\mu} a_{\mu}^{\dagger}, p_{\mu} a_{\mu}\right)\left(\begin{array}{c}
\mathbb{1} \\
a_{\mu-1}^{\dagger}
\end{array}\right)
\end{aligned}
$$


Equations (45) and (46) are to be read as a scalar product in an auxiliary space (see below). On inserting (45) into (39), $\hat{T}_{F}$ becomes

$$
\hat{T}_{F}=\left(\mathbb{1}, a_{M}^{\dagger}\right)\left[\prod_{\mu=1}^{M-1}\left(\begin{array}{cc}
\mathbb{1}-p_{\mu} a_{\mu} a_{\mu}^{\dagger} & a_{\mu}^{\dagger}-p_{\mu} a_{\mu} a_{\mu}^{\dagger} a_{\mu}^{\dagger} \\
p_{\mu} a_{\mu} & p_{\mu} a_{\mu} a_{\mu}^{\dagger}
\end{array}\right)\right]\left(\begin{array}{c}
\mathbb{1}-p_{M} a_{M} a_{M}^{\dagger} \\
p_{M} a_{M}
\end{array}\right)
$$

which may be rewritten as

$$
\hat{T}_{F}=\operatorname{Trace}\left[\prod_{\mu=1}^{M-1}\left(\begin{array}{cc}
\mathbb{1}-p_{\mu} a_{\mu} a_{\mu}^{\dagger} & a_{\mu}^{\dagger}-p_{\mu} a_{\mu} a_{\mu}^{\dagger} a_{\mu}^{\dagger} \\
p_{\mu} a_{\mu} & p_{\mu} a_{\mu} a_{\mu}^{\dagger}
\end{array}\right)\left(\begin{array}{cc}
\mathbb{1}-p_{M} a_{M} a_{M}^{\dagger} & \left(1-p_{M}\right) a_{M}^{\dagger} \\
p_{M} a_{M} & p_{M} \mathbb{1}
\end{array}\right)\right]
$$

To obtain (48) from (47) it may be checked that for arbitrary commuting operators $x_{i}$ and $y_{i}$ and arbitrary operators $z_{i}$, one has the identity

$$
\left(x_{1}, x_{2}\right) \quad\left(\begin{array}{ll}
y_{1} & y_{2} \\
y_{3} & y_{4}
\end{array}\right)\left(\begin{array}{l}
z_{1} \\
z_{2}
\end{array}\right)=\operatorname{Trace}\left[\left(\begin{array}{ll}
y_{1} & y_{2} \\
y_{3} & y_{4}
\end{array}\right)\left(\begin{array}{ll}
x_{1} z_{1} & x_{2} z_{1} \\
x_{1} z_{2} & x_{2} z_{2}
\end{array}\right)\right] .
$$

We stress here that (48) is merely a convenient way of writing the sums of products of the operators $\mathbb{1}, a_{\mu}, a_{\mu}^{\dagger}$ which form the transfer matrix. The two by two matrices appearing in (48), whose elements are made up of the operators $\mathbb{1}, a_{\mu}, a_{\mu}^{\dagger}$ may be thought of as acting in some auxiliary space. The trace is carried out in this auxiliary space and not in the space in which the operators $\mathbb{1}, a_{\mu}, a_{\mu}^{\dagger}$ act.

In a similar fashion one can construct the transfer matrix for backward updating, this time using (46) in (40). One obtains

$\hat{T}_{B}=\operatorname{Trace}\left[\left(\begin{array}{cc}\mathbb{1}-p_{M} a_{M} a_{M}^{\dagger} & p_{M} a_{M} \\ a_{M}^{\dagger}-p_{M} a_{M} a_{M}^{\dagger} a_{M}^{\dagger} & p_{M} a_{M} a_{M}^{\dagger}\end{array}\right) \prod_{\mu=1}^{M-1}\left(\begin{array}{cc}\mathbb{1}-p_{M-\mu} a_{M-\mu} a_{M-\mu}^{\dagger} & p_{M-\mu} a_{M-\mu} \\ \left(1-p_{M-\mu}\right) a_{M-\mu}^{\dagger} & p_{M-\mu} \mathbb{1}\end{array}\right)\right]$

Finally, to construct the transfer matrix for parallel dynamics $\hat{T}_{P}$ one should first realise that it is closely related to $\hat{T}_{F}$ since for forward updating each particle is unaffected by the results of previous updates in the sequence, except for the final particle $M$. After a little reflection it can be confirmed that

$$
\hat{T}_{P}=\operatorname{Trace}\left[\prod_{\mu=1}^{M}\left(\begin{array}{cc}
1-p_{\mu} a_{\mu} a_{\mu}^{\dagger} & a_{\mu}^{\dagger}-p_{\mu} a_{\mu} a_{\mu}^{\dagger} a_{\mu}^{\dagger} \\
p_{\mu} a_{\mu} & p_{\mu} a_{\mu} a_{\mu}^{\dagger}
\end{array}\right)\right]
$$

\section{2. Proof of the Steady State}

It was stated in section 2.1 that the steady state is given by (2), with $f_{\mu}\left(n_{\mu}\right)$ obeying (4) for parallel dynamics, (5) for forward sequential dynamics and (6) for backward sequential dynamics. The proof is quite similar in all three cases. We shall describe it in detail first 
for the case of parallel dynamics where the transfer matrix has the form (51). At the end of the subsection we shall return to forward sequential then backward sequential dynamics.

We first note that the vector space spanned by $\left|n_{1}, \ldots, n_{\mu}\right\rangle$ is a tensor product of $M$ spaces each with basis vectors $\mid n_{\mu}>$ where $n_{\mu}=0 \cdots \infty$

$$
\left|n_{1}, \ldots, n_{\mu}\right\rangle=\left|n_{1}>\otimes\right| n_{2}>\cdots \otimes \mid n_{M}>
$$

Thus the action of the transfer matrix for parallel dynamics (51) on (52) may be written as

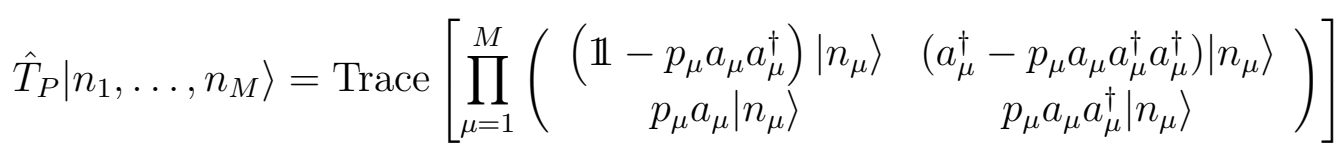

where the product, in fact, indicates a tensor product in the configuration space (as well as a usual product in the auxiliary space of two by two matrices) and the operators $\mathbb{1}, a_{\mu}, a_{\mu}^{\dagger}$ act on the space spanned by $\left|n_{\mu}\right\rangle$. To keep the notation light we have not explicitly indicated these matters, but the meaning is clear.

We are now in a position to prove the expression for the steady state given by (4) and (2). If the steady state is of the factorised form (2) we have

$$
\langle F|=\sum_{\left\{n_{\mu}\right\}}\left[f_{1}\left(n_{1}\right)\left\langle n_{1}\right|\right] \otimes\left[f_{2}\left(n_{2}\right)\left\langle n_{2}\right|\right] \cdots \otimes\left[f_{M}\left(n_{M}\right)\left\langle n_{M}\right|\right]
$$

and one finds from (53,54) that

$$
\left\langle F\left|\hat{T}_{P}\right| n_{1}, \ldots, n_{M}\right\rangle=\text { Trace }\left[\prod_{\mu=1}^{M} B_{\mu}\left(n_{\mu}\right)\right]
$$

where

$$
\begin{aligned}
B_{\mu}(0) & =\left(\begin{array}{cc}
f_{\mu}(0) & 0 \\
p_{\mu} f_{\mu}(1) & 0
\end{array}\right) \\
B_{\mu}(1) & =\left(\begin{array}{cc}
\left(1-p_{\mu}\right) f_{\mu}(1) & f_{\mu}(0) \\
p_{\mu} f_{\mu}(2) & p_{\mu} f_{\mu}(1)
\end{array}\right) \\
\text { and for } n>1 \quad B_{\mu}(n) & =\left(\begin{array}{cc}
\left(1-p_{\mu}\right) f_{\mu}(n) & \left(1-p_{\mu}\right) f_{\mu}(n-1) \\
p_{\mu} f_{\mu}(n+1) & p_{\mu} f_{\mu}(n)
\end{array}\right) .
\end{aligned}
$$

Inserting the expressions for $f_{\mu}(n)$ given in (4), and employing the Heaviside function we may rewrite (56 58) as

$$
B_{\mu}(n)=f_{\mu}(n) A_{\mu}(n)
$$

where

$$
A_{\mu}(n)=\left(\begin{array}{cc}
1-p_{\mu} \theta(n) & p_{\mu} \theta(n) \\
1-p_{\mu} \theta(n) & p_{\mu} \theta(n)
\end{array}\right)
$$


To prove the steady state (2) we must show that $\left\langle F\left|\hat{T}_{P}\right| n_{1}, \ldots, n_{M}\right\rangle=\prod_{\mu=1}^{M} f_{\mu}\left(n_{\mu}\right)$. Thus, on inserting (59) into (55), we see that it remains to show

$$
\text { Trace }\left[\prod_{\mu=1}^{M} A_{\mu}\left(n_{\mu}\right)\right]=1
$$

In order to do this we seek a similarity transformation

$$
\tilde{A}_{\mu}(n)=L_{\mu} A_{\mu}(n) R_{\mu+1} \quad \text { with } \quad R_{\mu} L_{\mu}=\mathbb{1}
$$

that puts $A_{\mu}(n)$ into the form

$$
\tilde{A}_{\mu}(n)=\left(\begin{array}{ll}
1 & x \\
0 & 0
\end{array}\right)
$$

where $x$ is any number. The trace of a product of such matrices (63) is clearly unity thus satisfying (61). A similarity transformation which fulfills (62, 63) is straightforward to construct. Taking

$$
R_{\mu}=\left(\begin{array}{cc}
1 & p_{\mu} \\
1 & -\left(1-p_{\mu}\right)
\end{array}\right) \quad \text { and } \quad L_{\mu}=\left(\begin{array}{cc}
1-p_{\mu} & p_{\mu} \\
1 & -1
\end{array}\right),
$$

it is easy to verify that $R_{\mu} L_{\mu}=\mathbb{1}$ and

$$
\tilde{A}_{\mu}(n)=L_{\mu} A_{\mu}(n) R_{\mu+1}=\left(\begin{array}{cc}
1 & p_{\mu+1}-p_{\mu} \theta(n) \\
0 & 0
\end{array}\right)
$$

hence (4) is proven.

In a similar manner the steady state for forward updating may be proven. Here we only provide a few key points. Following the steps (53) through to (60), now using (48) as the transfer matrix one finds that (60) holds for $\mu<M$ and

$$
A_{M}(n)=\left(\begin{array}{cc}
1-p_{M} \theta(n) & p_{M} \theta(n) \\
1-p_{M} & p_{M}
\end{array}\right)
$$

The similarity transformation (62) then gives

$$
\tilde{A}_{M}(n)=\left(\begin{array}{cc}
1 & p_{1}-p_{M}^{2}-p_{M}\left(1-p_{M}\right) \theta(n) \\
0 & p_{M}(1-\theta(n))
\end{array}\right)
$$

whilst the other $\tilde{A}_{\mu}(n)$ have the form (63). The trace of a product of these $\tilde{A}_{\mu}(n)$ again gives unity.

For backward updating, using (50) for the transfer matrix, one obtains for $\mu<M$

$$
A_{\mu}(n)=\left(\begin{array}{cc}
1-p_{\mu} \theta(n) & 1-p_{\mu} \\
p_{\mu} \theta(n) & p_{\mu}
\end{array}\right)
$$


and for $\mu=M$

$$
A_{M}(n)=\left(\begin{array}{cc}
1-p_{M} \theta(n) & 1-p_{M} \theta(n) \\
p_{M} \theta(n) & p_{M} \theta(n)
\end{array}\right)
$$

A suitable similarity transform is now

$$
\tilde{A}_{\mu}\left(n_{\mu}\right)=R_{\mu+1}^{\mathrm{T}} A_{\mu}\left(n_{\mu}\right) L_{\mu}^{\mathrm{T}}
$$

where $R_{\mu}, L_{\mu}$ are still given by (64). One then obtains for $\mu<M$

$$
\tilde{A}_{\mu}(n)=\left(\begin{array}{cc}
1 & 0 \\
p_{\mu+1}-p_{\mu}^{2}-p_{\mu}\left(1-p_{\mu}\right) \theta(n) & p_{\mu}(1-\theta(n))
\end{array}\right)
$$

and

$$
\tilde{A}_{M}(n)=\left(\begin{array}{cc}
1 & 0 \\
p_{1}-p_{M} \theta(n) & 0
\end{array}\right) .
$$

It is easy to convince oneself that the trace of a product of matrices of form (71) with a single matrix of form (72) yields unity, thus completing the proof of the steady state for backward updating.

\section{Generalisation to hopping probabilities dependent on empty space in front of particles}

In this section we consider hopping probabilities that depend on the number of empty sites in front of a particle. For this dynamics with random sequential updating, it is known that the steady state is given by a product measure [44]. Here for the disordered case we restrict our attention to parallel updating although it is straightforward to generalise to ordered sequential updating. Thus for parallel updating, at each time-step particle $\mu$ will hop forward with probability $p_{\mu}\left(n_{\mu}\right)$, where we recall $n_{\mu}$ is the number of empty sites in front of particle $\mu$ so that clearly $p_{\mu}(0)=0$. In a traffic model suitably chosen hopping probabilities could mimic the effect of deceleration when another car is encountered.

It turns out that the steady state again has the form (2). It will be shown in this section that

$$
\begin{aligned}
f_{\mu}(n) & =\left(1-p_{\mu}(1)\right) \text { for } n=0 \\
& =\frac{1-p_{\mu}(1)}{1-p_{\mu}(n)} \prod_{k=1}^{n} \frac{1-p_{\mu}(k)}{p_{\mu}(k)} \text { for } n>0 .
\end{aligned}
$$

In order to prove (73) we generalise the operators used in (41 44) by defining new operators $a_{\mu}(n), b_{\mu}(n)$ :

$$
\begin{aligned}
a_{\mu}(n) \mid n_{1}, \ldots, n_{\mu}, \ldots, n_{M}> & =\mid n_{1}, \ldots, n_{\mu}+1, \ldots, n_{M}>\quad \text { if } n_{\mu}=n \\
& =0 \text { otherwise } \\
b_{\mu}(n) \mid n_{1}, \ldots, n_{\mu}, \ldots, n_{M}> & =\mid n_{1}, \ldots, n_{\mu}, \ldots, n_{M}>\quad \text { if } n_{\mu}=n \\
& =0 \text { otherwise. }
\end{aligned}
$$


The corresponding operator to (41) is now given by

$$
h_{\mu-1 \mu}=\mathbb{1}-\sum_{n=1}^{\infty} p_{\mu}(n) b_{\mu}(n)+a_{\mu-1}^{\dagger} \sum_{n=1}^{\infty} p_{\mu}(n+1) a_{\mu}(n),
$$

where $a_{\mu-1}^{\dagger}$ is still defined by (43,44). Using the same procedure outlined in section 4 the transfer matrix (for parallel dynamics) may be constructed as

$$
\hat{T}_{P}=\operatorname{Trace}\left[\prod_{\mu=1}^{M}\left(\begin{array}{cc}
\mathbb{1}-\sum_{n=1}^{\infty} p_{\mu}(n) b_{\mu}(n) & a_{\mu}^{\dagger}-\left[\sum_{n=1}^{\infty} p_{\mu}(n) b_{\mu}(n)\right] a_{\mu}^{\dagger} \\
\sum_{n=1}^{\infty} p_{\mu}(n+1) a_{\mu}(n) & {\left[\sum_{n=1}^{\infty} p_{\mu}(n+1) a_{\mu}(n)\right] a_{\mu}^{\dagger}}
\end{array}\right)\right] .
$$

Using this transfer matrix and assuming that the steady state is of the form (2), in analogy with section 4 one arrives at (55) where now

$$
B_{\mu}(n)=\left(\begin{array}{cc}
f_{\mu}(n)\left(1-p_{\mu}(n)\right) & f_{\mu}(n-1)\left(1-p_{\mu}(n-1)\right) \theta(n) \\
f_{\mu}(n+1) p_{\mu}(n+1) & f_{\mu}(n) p_{\mu}(n)
\end{array}\right) .
$$

and it should be borne in mind that $p_{\mu}(0)=0$. Inserting (73) one finds that it remains to show condition (61) is satisfied where this time

$$
A_{\mu}(n)=\left(\begin{array}{ll}
1-p_{\mu}(n) & p_{\mu}(n) \\
1-p_{\mu}(n) & p_{\mu}(n)
\end{array}\right)
$$

It is easy to check that inserting $R_{\mu}\left(n_{\mu}\right) L_{\mu}\left(n_{\mu}\right)(=\mathbb{1})$ before each $A_{\mu}\left(n_{\mu}\right)$ in the product (61), where

$$
R_{\mu}\left(n_{\mu}\right)=\left(\begin{array}{cc}
1 & p_{\mu}\left(n_{\mu}\right) \\
1 & -\left(1-p_{\mu}\left(n_{\mu}\right)\right)
\end{array}\right) \quad \text { and } \quad L_{\mu}(n)=\left(\begin{array}{cc}
1-p_{\mu}\left(n_{\mu}\right) & p_{\mu}\left(n_{\mu}\right) \\
1 & -1
\end{array}\right)
$$

yields the required similarity transformation.

\section{Conclusion}

In this paper it has been shown that analytical results may be obtained for the asymmetric exclusion model with parallel dynamics which forms the basis for many discrete models of traffic flow. Ordered sequential dynamics have also been treated using similar techniques. Previously, the steady state for the situation where at each parallel time-step each particle attempts forward with probability $p$ had been solved [8]. Here we have generalised that model to one where at each time-step each particle $\mu$ attempts a hop forward with probability $p_{\mu}\left(n_{\mu}\right)$, an arbitrary function of $n_{\mu}$ the number of empty sites immediately ahead of particle $\mu$. The method used to prove the steady state (73) was to construct the transfer matrix using a technique inspired by [39]. 
A detailed analysis was made of the case where $p_{\mu}$ did not depend on $n_{\mu}$, and it was shown that a phase transition analogous to Bose condensation occurs, as it did for a random sequential updating [37]. In particular it is interesting to note equation (12) which shows the velocity in the particle system is intimately related to the fugacity (or equivalently chemical potential) when the system is viewed as a Bose system. Since in (single component) equilibrium systems the thermodynamic phase is determined by minimising the chemical potential, the suggestion is that in simple traffic flow models a principle of minimisation of velocity pertains.

A simple illustration of this principle is the example of a single slow particle with hopping rate $c$ while all the other particles have hopping rate $p$ [37. Then in a phase where there is a tailback behind the slow particle and empty space in front, the velocity is $c$ whereas in a congested phase the velocity should be given by $(1-\sqrt{1-4 p \rho(1-\rho)}) /(2 \rho)[8$. Choosing the phase with the minimum velocity yields the critical density $\rho_{c}=(p-c) /\left(p-c^{2}\right)$. This expression is, in fact, exact as can be checked by using the results of section 3 . It is important to ascertain whether a principle of minimisation of velocity holds in more complicated traffic flow models.

It was also interesting to note that parallel dynamics and forward sequential dynamics have the same thermodynamic behaviour e.g. the same critical density. One expects this since a time-step of forward updating and a parallel time-step only differ in last update of the sequence in forward dynamics. However backward updating has a distinct critical density as does random sequential updating. Comparing the three critical densities reveals that the highest is for backward dynamics, the lowest is for random sequential dynamics and parallel dynamics lies in between. This confirms that backward dynamics is the most efficient updating scheme in terms of throughput and random sequential is the worst.

It would be interesting to explore other realisations for the case of general $p_{\mu}\left(n_{\mu}\right)$ considered in section 5. In particular it should be possible to analyse the effect of a braking distance for each particle.

The present work narrows the gap between bona fide models of traffic flow, such as that of Nagel and Schreckenberg or more sophisticated models [5], and the simple particle hopping models for which exact results are possible. In order to close the gap further, analytical results are desirable for models where particles can hop more than one lattice site at each update. Some progress has already been made towards this [30].

It would also be of interest to analyse more complicated properties of the system such as relaxation to the steady state, as studied numerically in [38, 41], or else the diffusion constants of particles [17, 18].

\section{Acknowledgments}

I thank F. H. L. Essler, H. Hinrichsen and G. M. Schütz for useful discussions and especially $\mathrm{V}$. Hakim for patient explanation of the technique used in [39]. 


\section{Appendix A: Evaluation of the Integral (20)}

In this appendix we derive the expansion (23 25) of the integral (20) given by

$$
I(v)=\frac{\gamma+1}{(1-c)^{\gamma+1}} \int_{c}^{1} d p \frac{v(p-c)^{\gamma}}{p-v} \text {. }
$$

By first defining

$$
v=c \exp (-\epsilon)
$$

(A1) becomes

$$
I(\epsilon)=\frac{\gamma+1}{(1-c)^{\gamma+1}} \int_{0}^{1-c} d p \frac{c \exp (-\epsilon) p^{\gamma}}{p+c-c \exp (-\epsilon)}
$$

and we may follow an analysis similar to that of Robinson [43. We make the Mellin transformation

$$
\mathcal{I}(s)=\int_{0}^{\infty} d \epsilon I(\epsilon) \epsilon^{s-1}
$$

which may be expanded to yield

$$
\mathcal{I}(s)=\frac{\gamma+1}{(1-c)^{\gamma+1}} \int_{0}^{1-c} d p p^{\gamma} \sum_{n=0}^{\infty}\left(\frac{c}{p+c}\right)^{n+1} \frac{\Gamma(s)}{(n+1)^{s}},
$$

where $\Gamma(s)$ is the usual Gamma function defined by

$$
\Gamma(s)=\int_{0}^{\infty} d \epsilon \exp (-\epsilon) \epsilon^{s-1}
$$

The inverse transformation is

$$
I(\epsilon)=\frac{1}{2 \pi i} \int_{x-i \infty}^{x+i \infty} d s \mathcal{I}(s) \epsilon^{-s}
$$

where $x$ is a real constant chosen so that the contour of integration is to the right of any pole. The integral can be evaluated by closing the contour and using the calculus of residues. The analytic structure of

$$
\mathcal{I}(s)=g_{\gamma}(s) \Gamma(s)
$$

where

$$
g_{\gamma}(s)=\frac{\gamma+1}{(1-c)^{\gamma+1}} \int_{0}^{1-c} d p p^{\gamma} \sum_{n=0}^{\infty}\left(\frac{c}{p+c}\right)^{n+1} \frac{1}{(n+1)^{s}},
$$

is as follows: $\Gamma(s)$ has simple poles at $s=-n$ where $n=0,1, \ldots \infty$ with residues $(-1)^{n} / n$ ! and $g_{\gamma}(s)$ has a simple pole at $s=-\gamma$. To evaluate the residue of $g_{\gamma}(s)$ at $s=-\gamma$ we note the small $p$ behaviour of the sum involved in (A9)

$$
\sum_{n=0}^{\infty}\left(\frac{c}{p+c}\right)^{n+1} \frac{1}{(n+1)^{s}}
$$


Thus, one obtains

$$
\lim _{s \rightarrow-\gamma}\left[g_{\gamma}(s)(s+\gamma)\right]=(1+\gamma)\left(\frac{c}{1-c}\right)^{1+\gamma} \Gamma(1+\gamma)
$$

and

$$
I(\epsilon)=(1+\gamma)\left(\frac{c}{1-c}\right)^{1+\gamma} \Gamma(1+\gamma) \Gamma(-\gamma) \epsilon^{\gamma}+\sum_{n=0}^{\infty} \frac{g_{\gamma}(-n)}{n !}(-\epsilon)^{n} .
$$

The expansion $(23,25)$ is the first two terms in (A12) where it can be computed that

$$
\begin{aligned}
g_{\gamma}(0) & =\frac{1+\gamma}{\gamma} \frac{c}{1-c} \\
g_{\gamma}(-1) & =\frac{1+\gamma}{\gamma} \frac{c}{1-c}\left[1+\frac{\gamma}{\gamma-1} \frac{c}{1-c}\right] .
\end{aligned}
$$

For $\gamma \rightarrow 1$, the singularity in (A14) cancels with the singularity in the first term in the rhs of (A12) and one obtains

$$
\begin{aligned}
I(\epsilon) & =g_{\gamma}(0)+\lim _{\gamma \rightarrow 1}\left[(1+\gamma)\left(\frac{c}{1-c}\right)^{1+\gamma} \Gamma(1+\gamma) \Gamma(-\gamma) \epsilon^{\gamma}-g_{\gamma}(-1) \epsilon\right]+\ldots \\
& =g_{\gamma}(0)+2\left(\frac{c}{1-c}\right)^{2} \epsilon \ln \epsilon+\mathcal{O}(\epsilon) .
\end{aligned}
$$




\section{References}

[1] B. Schmittmann and R K P Zia (1995) Statistical Mechanics of Driven Diffusive Systems vol. 17 of Domb and Lebowitz series, Academic Press, U.K.

[2] H. Spohn (1991) Large Scale Dynamics of Interacting Particles (Springer-Verlag, New York)

[3] For a recent review see B. Derrida and M. R. Evans chapter 14 in Nonequilibrium Statistical Mechanics in One Dimension (1997), ed V. Privman, Cambridge University Press and references therein.

[4] O. Biham, A. A. Middleton and D. Levine (1992) Phys. Rev. A 466124

[5] K.Nagel (1996) Phys. Rev. E 534655 and references therein

[6] K.Nagel and M. Schreckenberg (1992) J.Phys. (France) I 22221

[7] S. Migowsky, T. Wanschura, P. Ruján (1994) Z. Phys. B 95407

[8] M. Schreckenberg, A. Schadschneider, K. Nagel and M. Ito (1995) Phys. Rev. E 51 2339

[9] T. Nagatani (1995) Phys. Rev. E 51922

[10] T. Nagatani (1995) J. Phys. A 28 L119

[11] K. Nagel and M. Paczuski (1995) Phys. Rev. E 512909

[12] G. Csányi and J. Kertész (1995) J. Phys. A 28 L427

[13] L H Gwa and H Spohn (1992) Phys. Rev. A46 844

[14] B. Derrida, M. R. Evans, V. Hakim and V. Pasquier (1993) J. Phys. A 261493

[15] G. Schütz and E. Domany (1993) J. Stat. Phys. 72277

[16] J.M.J van Leeuwen and A. Kooiman (1992) Physica A 18479

[17] B. Derrida, M. R. Evans and D. Mukamel (1993) J. Phys. A 264911

[18] B. Derrida, M. R. Evans and K. Mallick (1995) J. Stat. Phys. 79833

[19] G. M. Schütz (1997) preprint cond-mat/9701019

[20] B. Derrida, S.A. Janowsky, J.L. Lebowitz, E.R. Speer (1993) Europhys. Lett. 22651 
[21] S. Sandow (1994) Phys. Rev. E 502660

[22] M. R. Evans, D. P. Foster, C. Godrèche, D. Mukamel (1995) J. Stat. Phys. 8069

[23] F. H. L. Essler and V. Rittenberg (1996) J. Phys. A 293375

[24] B. Derrida, J.L. Lebowitz and E.R. Speer (1997) J. Stat. Phys. to appear

[25] G. Schütz (1993) Phys. Rev. E 474265

[26] H. Hinrichsen (1996) J. Phys. A 293659

[27] H. Hinrichsen and S. Sandow (1997) J. Phys. A 302745

[28] N. Rajewsky, A. Schadschneider and M. Schreckenberg (1996) J. Phys. A 29 L305

[29] A. Honecker and I. Peschel (1996) preprint cond-mat/9606053

[30] J.G. Brankov, V.B. Priezzhev, A. Schadschneider and M. Schreckenberg (1996) J. Phys. A 29 L229

[31] S.A. Janowsky and J.L. Lebowitz (1992) Phys. Rev. A 45618

[32] Z. Csahók and T. Vicsek (1994) J. Phys. A 27 L591

[33] Z. Toroczkai and R. K. P. Zia (1996) Phys. Lett. A 21797

[34] B. Derrida (1996) in Statphys-19 (19th IUPAP International Conference on Statistical Physics, Xiamen) ed. B-L Hao, World Scientific, Singapore

[35] K. Mallick (1996) J. Phys. A 295375

[36] I. Benjamini, P. A. Ferrari, C. Landim (1996) Stoch. Proc. Appl. 61181

[37] M. R. Evans (1996) Europhys. Lett. 3613

[38] J. Krug and P. A. Ferrari (1996) J. Phys. A 29 L465

[39] V. Hakim and J P Nadal (1983) J. Phys. A 16 L213

[40] E. Ben-Naim, P. L. Krapivsky and S Redner (1994) Phys. Rev. E 50822

[41] D. V. Ktitarev, D. Chowdhury and D. E. Wolf (1997) J. Phys. A 30 L221

[42] For a clear exposition see e.g. K. Huang Statistical Mechanics 2nd edition (1987) (Wiley, New York)

[43] J. E. Robinson (1951) Phys. Rev. 83678

[44] F. Spitzer (1970) Adv. in Math. 5246 